\title{
A CRITERION FOR CELLULARITY IN A MANIFOLD. II
}

\author{
BY \\ D. R. McMilLAN, JR.( $\left.{ }^{1}\right)$
}

1. Introduction. Let $X$ be a compact absolute retract in the interior of a piecewise-linear (abbreviated: pwl) $n$-manifold $M^{n}$. We say that $X$ is cellular with respect to pwl cells in $M^{n}$ if there exists a sequence $F_{1}, F_{2}, \ldots$ of pwl $n$-cells in $M^{n}$ such that $F_{i+1} \subset$ Int $F_{i}$ and $X=\bigcap_{i=1}^{\infty} F_{i}$. In [12] we showed that, if $n \geqq 5$, then $X$ is cellular with respect to pwl cells in $M^{n}$ if and only if: for each open set $U$ in $M^{n}$ such that $X \subset U$, there is an open set $V$ in $M^{n}$ such that $X \subset V \subset U$ and each loop in $V-X$ is contractible in $U-X$. In case $n=3$ and this condition holds, the proofs of Lemma 1 and Theorem 1' of [12] yield only the unsatisfactory result that $X$ is the intersection of a sequence $F_{1}, F_{2}, \ldots$ of homotopy 3-cells, where $F_{i+1} \subset$ Int $F_{i}$.

A corollary of the theorems presented here is that if, in the case $n=3, X$ is homeomorphic to a collapsible complex (see [15]), then $X$ is cellular if and only if the above cellularity criterion holds. In particular, our results apply to closed topological cells of dimension three or less. The problem is to show that some neighborhood of $X$ in $M^{3}$ is embeddable in $E^{3}$. If $M^{3}$ is orientable, this reduces to the question of whether polygonal arcs can be deformed away from $X$ with a homeomorphism of $M^{3}$.

By a cube-with-handles we mean any complex piecewise-linearly equivalent to the regular neighborhood in $E^{3}$ of a finite graph. If $X$ is a compact subset of Int $M^{3}$, then $X$ has the cube-with-handles property if $X=\bigcap_{i=1}^{\infty} H_{i}$, where $H_{i}$ is a polyhedral cube-with-handles in $M^{3}$ and $H_{i+1} \subset$ Int $H_{i}$. It follows from our Corollary 1.3 that collapsible complexes have the cube-with-handles property under every topological embedding in the interior of a 3-manifold. The same is true of 2-manifolds with nonempty boundary, if the 3-manifold $M^{3}$ is orientable (see the Remark following Corollary 1.3). We regard Theorem 1 and Corollary 1.2 as our main results, although Corollary 1.3 is perhaps most useful for verifying that subsets have the cube-with-handles property.

In general, we shall use the terminology of [12]. In particular, we retain the convention that a manifold is always connected. All of the manifolds considered will be assumed to have a fixed pwl (i.e., combinatorial) structure. A closed manifold is compact and without boundary. An open manifold is noncompact and without boundary. By an $n$-frame $(n \geqq 2)$ we mean any space homeomorphic to the join of

Presented to the Society, November 27, 1965 under the title Canonical neighborhoods in 3manifolds; received by the editors November 3, 1965.

( $\left.{ }^{1}\right)$ This research was supported in part by grant NSF GP-4125. The author is an Alfred P. Sloan Fellow. 
a point with $n$ other distinct points. A null sequence $X_{1}, X_{2}, \ldots$, of subsets of a metric space is one such that the diameters of the $X_{i}$ 's converge to zero.

Our results lean heavily on the papers of R. H. Bing listed in the references. The reader is referred to $[4, \S 3]$ for a discussion of Sierpiński curves. A Sierpiński curve in the interior of a 3-manifold $M^{3}$ is said to be tame if it lies on a tame 2-sphere in $M^{3}$. With this understanding, the proofs of Theorems 8.2 and 9.1 of [6] can be adapted to cover the case of a 2 -sphere embedded in the interior of a 3-manifold (see [7]).

Finally, we wish to thank R. H. Bing for many helpful discussions concerning the results given here.

2. Tunneling into three-manifolds. The following lemma tells us how to bore holes in an orientable 3-manifold-with-boundary so as to obtain a cube-withhandles.

LEMMA 1. Let $N$ be a compact, orientable piecewise-linear 3-manifold with nonempty boundary. Then $N$ contains a finite, disjoint collection of polygonal arcs $A_{1}, A_{2}, \ldots, A_{k}$, such that $A_{i} \cap \mathrm{Bd} N=\mathrm{Bd} A_{i}$, for each $i$, and the closure of $N$ minus a sufficiently small regular neighborhood of each $A_{i}$ is a cube-with-handles.

Proof. Since $\mathrm{Bd} N$ is nonempty, there is a 2-complex $K \subset N$ such that $N$ contracts (in the sense of J. H. C. Whitehead [15]) to $K$. We may obtain a 3-manifold equivalent to a regular neighborhood of $K$ in $N$ by attaching, in some manner, disjoint polyhedral 3-cells $B_{1}, B_{2}, \ldots, B_{k}$ (essentially, obtained by thickening the 2simplexes of $K$ ) to a cube-with-handles $H$ (a small regular neighborhood of the 1-skeleton of $K)$ in such a way that each $B_{i} \cap H=\left(\mathrm{Bd} B_{i}\right) \cap(\mathrm{Bd} H)$ is an annulus. Since the assertion of the lemma clearly holds for a 3-manifold of this form, and since $N$ is combinatorially equivalent to any regular neighborhood of $K$ in $N$, the result follows.

The following is an application of Lemma 1.

LEMMA 2. Let $X$ be a compact, connected subset of Int $M$, where $M$ is an orientable piecewise-linear 3-manifold. Then, $X$ has the cube-with-handles property if and only if the following condition holds:

For each polygonal arc $A \subset \mathrm{Int} M$ such that $X \cap \mathrm{Bd} A=\varnothing$, and for each $\varepsilon>0$, there is a homeomorphism $h$ of $M$ onto $M$ such that $h$ is the identity outside the $\varepsilon$-neighborhood of $X$, and $X \cap h(A)=\varnothing$.

Proof. Suppose first that the condition holds, and let $U$ be an open subset of Int $M$ containing $X$. Let $N$ be a compact, orientable piecewise-linear 3-manifold with nonempty boundary such that $X \subset$ Int $N \subset N \subset U$. Let $A_{1}, A_{2}, \ldots, A_{k}$, be the arcs promised by Lemma 1 . Then, by our condition, there is a homeomorphism $h_{1}: N \rightarrow N$ such that $h_{1}$ is the identity on a neighborhood of $\mathrm{Bd} N$ and $X \cap h_{1}\left(A_{1}\right)$ $=\varnothing$. By [1, Theorem 3], we may suppose that $h_{1}$ is pwl. Applying the condition inductively gives pwl homeomorphisms $h_{1}, h_{2}, \ldots, h_{k}$ of $N$ onto $N$ such that, for 
$2 \leqq i \leqq k, h_{i}$ is the identity on $\operatorname{Bd} N$ and on $h_{i-1} h_{i-2} \cdots h_{1}\left(A_{1} \cup \cdots \cup A_{i-1}\right)$, and $X \cap h_{i}\left[h_{i-1} \cdots h_{1}\left(A_{i}\right)\right]=\varnothing$. Then, $h=h_{k} h_{k-1} \cdots h_{1}$ is a pwl homeomorphism of $N$ onto $N$ such that $h$ is the identity on $\operatorname{Bd} N$, and $X \cap h\left(A_{i}\right)=\varnothing$, for all $i$. The closure of $N$ minus a small regular neighborhood of each $h\left(A_{i}\right)$ is a polyhedral cube-withhandles $H$ such that $X \subset$ Int $H \subset H \subset U$, and the proof is complete.

Now suppose that $X$ has the cube-with-handles property, and let $A$ and $\varepsilon>0$ be given. Let $H$ be a polyhedral cube-with-handles in the $\varepsilon$-neighborhood of $X$ such that $X \subset$ Int $H$ and $H \cap \operatorname{Bd} A=\varnothing$. Choose a one-dimensional polyhedron $G$ in (Int $H)-A$ such that $H$ contracts to $G$, and let $T$ be a "thin" regular neighborhood of $G$ in (Int $H$ ) $-A$. The desired homeomorphism $h$ is then one which expands $T$ until it exactly covers $H$, and is the identity outside a small neighborhood of $H$ (the closure of $H-T$ is topologically $(\mathrm{Bd} H) \times[0,1])$.

The following is probably well known, but we include its proof for completeness.

Lemma 3. Let $M$ be a piecewise-linear 3-manifold, $A$ a tame arc in $M$ with (Int $A) \cap(\operatorname{Bd} M)=\varnothing$, and $X$ a compact subset of Int $M$. Suppose also that $X$ is either zero-dimensional or a one-dimensional absolute neighborhood retract. Then for given $\varepsilon>0$, there is a homeomorphism $h: M \rightarrow M$ such that $A \cap h(X)=\varnothing, h$ moves each point less than $\varepsilon$, and $h$ is the identity outside the e-neighborhood of $X \cap A$.

Proof. There is no loss of generality in assuming that $X \cap A$ is a 0 -dimensional subset of Int $A$. Since $A$ is tame, the $\varepsilon$-neighborhood of $X \cap A$ contains a disjoint collection $C_{1}, C_{2}, \ldots, C_{m}$, of tame 3-cells whose interiors cover $X \cap A$, such that each $C_{j}$ has diameter less than $\varepsilon$ and $C_{j} \cap A$ is an unknotted spanning $\operatorname{arc}$ of $C_{j}$, for each $j$. In case $X$ is a 1-dimensional absolute neighborhood retract, we also suppose that the diameter of each $C_{j}$ is less than $\delta$, where $\delta$ is a positive number such that each subset of $X$ of diameter less than $\delta$ is contractible to a point in $X$.

Suppose $A \cap \mathrm{Bd} C_{j}=a_{j} \cup b_{j}$. It then follows from the way that the $C_{j}$ 's were selected that $X \cap \mathrm{Bd} C_{j}$ cannot separate $\mathrm{Bd} C_{j}$ (since $X \cap \mathrm{Bd} C_{j}$ is either a compact 0 -dimensional set or a compact 1-dimensional set each of whose components is acyclic in the sense of Čech homology). Let $B_{j}$ be an arc in $\left(B d C_{j}\right)-X$ from $a_{j}$ to $b_{j}$. The desired homeomorphism $h$ is then defined to be the identity outside a small neighborhood of each $C_{j}$, and to take each $B_{j}$ onto $C_{j} \cap A$, leaving $A-\bigcup$ Int $C_{j}$ fixed.

Corollary. Let $M$ be an orientable piecewise-linear 3-manifold and X a compact subset of Int $M$. If $X$ is either zero-dimensional or a one-dimensional absolute neighborhood retract, then for given $\varepsilon>0$, the $\varepsilon$-neighborhood of $X$ contains a finite disjoint collection of polyhedral cubes-with-handles whose interiors cover $X$.

Proof. This follows from Lemma 1, Lemma 3, and the first half of the proof of Lemma 2. In case $X$ is connected, the collection of cubes-with-handles need not contain more than one element, of course. 
3. Pushing arcs away from cells. For the reader's convenience, we quote two results from the literature before beginning the proof of Theorem 1 . The next lemma follows from [11].

LeMma 4. Let $D$ be a 2-cell topologically embedded in the interior of a 3-manifold $M$. Let $p \in \mathrm{Bd} D, q \in \operatorname{Int} D$, and $\varepsilon>0$. Then there is a tame arc $A$ in $D$ joining $a$ point $r \in \operatorname{Bd} D$ to a point $s \in \operatorname{Int} D$ such that $(\operatorname{Int} A) \cap(\operatorname{Bd} D)=\varnothing, r$ is within $\varepsilon$ of $p$, and $s$ is within $\varepsilon$ of $q$.

We shall also need the following useful result of Bing [8, Theorem 2]:

Lemma 5. Suppose $S$ is a topological 2-sphere and $A$ is a tame arc in $E^{3}$. Then for each $\varepsilon>0$ there is a homeomorphism $h: E^{3} \rightarrow E^{3}$ such that $A$ intersects $h(S)$ at only a finite number of points and pierces it at each of these points, $h$ moves each point less than $\varepsilon$, and $h$ is the identity outside the $\varepsilon$-neighborhood of $S \cap A$. Further, if $p \in A \cap h(S)$, there is an arc $Z$ in $S$ such that $p \in \operatorname{Int} Z$ and $A \cup Z$ is a tame 4-frame.

REMARK 1. This lemma extends immediately to the case of a finite number of mutually exclusive, tame arcs $A_{1}, A_{2}, \ldots, A_{k}$. We shall also need to use it when the 2-sphere $S$ is replaced by a 2-cell $C$. This is justified as follows. Use Lemma 3 to shift $C$ slightly until Bd $C$ misses each $A_{i}$. Another slight adjustment will make each $A_{i} \cap C$ a zero-dimensional subset of Int $C$. Finally, we use the above lemma and the fact that sufficiently small subdisks of Int $C$ can be extended to 2-spheres [2, Theorem 5], to get the desired result.

REMARK 2. There is a simple closed curve $J$ in Int $C$, and containing $h(C) \cap \bigcup A_{i}$, such that $J \cup \cup A_{i}$ is tame. Such a curve may be pieced together from the $Z$ 's promised by the last sentence of Lemma 5 and the arcs given by Lemma 4 . We show local tameness (and hence tameness, by [1, Theorem 9]) of $J \cup \cup A_{i}$ by appealing to the fact that the union of two tame arcs meeting only at an endpoint is tame if this union lies interior to a 2-cell (this follows from [10, Theorem 3] and [9, Theorem 1]).

REMARK 3. Similarly, if $h(C) \cap \bigcup A_{i}$ consists of the points $p_{1}, p_{2}, \ldots, p_{m}$, and $E$ is an arc in $\operatorname{Bd} h(C)$, there are mutually exclusive arcs $J_{1}, J_{2}, \ldots, J_{m}$, in $h(C)-E$ such that $\mathrm{Bd} J_{j}$ consists of $p_{j}$ plus the single point $J_{j} \cap \operatorname{Bd} h(C)$, for each $j$, and $\bigcup J_{j} \cup \cup A_{i}$ is tame.

THEOREM 1. Let $B$ be a $q$-cell $(q=1,2$, or 3$)$ topologically embedded in $E^{3}$, and let $D \subset \mathrm{Bd} B$ be $a(q-1)$-cell. Let $A_{1}, A_{2}, \ldots, A_{k}$ be a finite disjoint collection of tame arcs in $E^{3}-D$ with each $\mathrm{Bd} A_{i} \subset E^{3}-B$. Then, there exists a compact set $C \subset B-D$ such that, for given $\varepsilon>0$, there is a homeomorphism $h: E^{3} \rightarrow E^{3}$ with each $h\left(A_{i}\right)$ $\subset E^{3}-B$ and $h$ is the identity outside the $\varepsilon$-neighborhood of $C$.

Proof. Let $C$ be a topological $q$-cell in $B-D$ containing $B \cap \cup A_{i}$ and such that the closure of $B-C$ is a $q$-cell meeting $C$ in a $(q-1)$-cell $E$ missing $\bigcup A_{i}$. We assume 
that there is given a positive number $\varepsilon$ less than the distance from $C$ to $D$ and from $\cup A_{i}$ to the closure of $B-C$.

1. The case $q=1$. This follows immediately from Lemma 3 .

2. The case $q=2$. By the first remark following Lemma 5, we may assume without loss of generality that each $A_{i}$ has been adjusted slightly so that $B \cap \bigcup A_{i}$ is a finite set of points $p_{1}, p_{2}, \ldots, p_{m}$, in Int $C$ such that, for each $j$, there is some arc in $\bigcup A_{i}$ which pierces $B$ at $p_{j}$. By the third remark following Lemma 5 , there are mutually exclusive arcs $J_{1}, J_{2}, \ldots, J_{m}$, in $C-E$ such that $\mathrm{Bd} J_{j}$ consists of $p_{j}$ plus the single point $J_{j} \cap \mathrm{Bd} C$, for each $j$, and $\bigcup J_{j} \cup \cup A_{i}$ is tame.

In particular, since $J_{j}$ plus some arc $Y_{j}$ of diameter less than $\varepsilon$ in $\bigcup A_{i}$ is a tame 3-frame, there are mutually exclusive tame 3-cells $F_{1}, F_{2}, \ldots, F_{m}$, in the $\varepsilon$-neighborhood of $C$ and missing the closure of $B-C$ such that $Y_{j}=F_{j} \cap \bigcup A_{i}, J_{j} \subset \operatorname{Int} F_{j}$, and $Y_{j}$ is an unknotted spanning arc of $F_{j}$. Suppose Bd $Y_{j}=a_{j} \cup b_{j}$.

We claim that for each $j$ there is an $\operatorname{arc} R_{j}$ in $\mathrm{Bd} F_{j}$ joining $a_{j}$ and $b_{j}$, and missing $C$. If not, then some component $K$ of $C \cap \mathrm{Bd} F_{j}$ separates $a_{j}$ from $b_{j}$ in $\mathrm{Bd} F_{j}$.

If $L$ is a simple closed curve formed by joining $a_{j}$ and $b_{j}$ with an arc in $E^{3}-B$ whose interior misses $F_{j}$, then, since $K \subset C-\bigcup J_{j}$ and $C-\bigcup J_{j}$ is simply-connected, there is a neighborhood $U$ of $K$ in $E^{3}$ such that each simple closed curve in $U$ is contractible in $E^{3}-L$. But $U \cap \mathrm{Bd} F_{j}$ is a neighborhood of $K$ in $\mathrm{Bd} F_{j}$ and so must contain a simple closed curve separating $a_{j}$ from $b_{j}$ (since $K$ is the intersection of a monotonically decreasing sequence of punctured 2-cells in $\mathrm{Bd} F_{j}$ ), and this simple closed curve must link $L$ (in the homology sense), a contradiction. Hence, the arc $R_{j}$ exists, for each $j$.

The desired homeomorphism $h$ is now defined as one which is the identity outside a small neighborhood of each $F_{j}$, and which, for each $j$, takes $Y_{j}$ onto $R_{j}$ leaving $a_{j}$ and $b_{j}$ fixed.

3. The case $q=3$. Let $S=\mathrm{Bd} C$. By Lemma 5 , we may assume that each $A_{i}$ has been adjusted slightly so that $S \cap \bigcup A_{i}$ is a finite set of points $p_{1}, p_{2}, \ldots, p_{m}$, in $S-E$ and that, for each $j$, some arc in $\bigcup A_{i}$ pierces $S$ at $p_{j}$. By the second remark following Lemma 5 , if we let $F$ denote the closure of $S-E$, there is a simple closed curve $J \subset \operatorname{Int} F$ which contains $p_{1}, p_{2}, \ldots, p_{m}$, and such that $J \cup \cup A_{i}$ is tame.

By [6, Theorem 9.1], there is a tame Sierpiński curve $X$ in $S$ such that each point of $J$ is an inaccessible point of $X$. Let $S_{0}$ be a 2-sphere in the 3-cell $C$ homeomorphically close to $S$ such that $S_{0} \cap S=X, S_{0} \cap \bigcup A_{i}=S \cap \bigcup A_{i}$, and $S_{0}$ is locally tame except possibly at $X$ (intuitively, to obtain $S_{0}$ we deform $S$ into Int $C$ along the components of $S-X)$. By [6, Theorem 8.2], $S_{0}$ is tame, and hence has a cartesian product neighborhood $N$ (i.e., one homeomorphic to $S_{0} \times[-1,1]$, with $S_{0} \times\{0\}$ corresponding to $S_{0}$ ).

By [14, Theorem 1], there is a homeomorphism of $N$ onto $S_{0} \times[-1,1]$ as above with some open arc of $\bigcup A_{i}$ about each $p_{j}$ corresponding to the product of $p_{j}$ with some open interval about 0 . Thus there is a tame 2-sphere $S_{1}$ in $N$ such that $S_{1} \cap S_{0}=J$, the two components of $S_{1}-J$ are in different components of $N-S_{0}$, 
and $S_{1}$ contains the closure of an open arc of $\bigcup A_{i}$ about each $p_{j}$, and otherwise misses $\bigcup A_{i}$.

Note that $S_{1} \cap X=J$ and that each point of $J$ is an inaccessible point of $X$. Hence, if the components of $S-X$ are $K_{1}, K_{2}, \ldots$, then $S_{1} \cap S$ consists of $J$ plus the null sequence of compact sets: $S_{1} \cap K_{1}, S_{1} \cap K_{2}, S_{1} \cap K_{3}, \ldots$. Since each $S_{1} \cap K_{i}$ can be covered by the interiors of a finite number of disjoint 2-cells lying in $S_{1}$ and having boundaries close to $S_{1} \cap K_{i}$, it follows that there is a disjoint, null sequence of 2-cells in $S_{1}-J$ whose interiors cover $S_{1} \cap(S-J)$. Thus, there is a (tame) 2-cell $G$ in $S_{1}$ within $\varepsilon / 2$ of $J$ and missing the closure of $B-C$ such that $G \cap J$ is a spanning arc of $G$ containing $p_{1}, \ldots, p_{m}$ in its interior, the two components of $(\mathrm{Bd} G)-J$ are in different components of $E^{3}-S$, and $G \cap \bigcup A_{i}$ is a collection of spanning arcs of $G$, each joining one component of $(\mathrm{Bd} G)-J$ to the other.

Let $h_{1}: E^{3} \rightarrow E^{3}$ be a homeomorphism which is the identity outside a small neighborhood of the topological half plane (Int $G)-($ Int $C)$ and such that, for some neighborhood $U$ of the open arc Int $(G \cap J), h_{1}(U \cup[(\operatorname{Int} G)-C]) \subset E^{3}-C$. We may think of $h_{1}$ as squeezing the 2-cell $G-\operatorname{Int} C$ very close to the $\operatorname{arc}(\operatorname{Bd} G)-\operatorname{Int} C$, while keeping this arc fixed. Now, using the fact that $C$ is a 3-cell, let $h_{2}: E^{3} \rightarrow E^{3}$ be a homeomorphism which is the identity on $E^{3}-$ Int $C$ and such that

$$
h_{2}\left(C \cap \bigcup A_{i}\right) \subset U \text {. }
$$

The required homeomorphism is then defined to be $h=h_{1} h_{2}$.

CoRollaRY 1.1. Let $K$ be a finite complex and $L$ a subcomplex of $K$ such that $K$ contracts to $L$. Let $M$ be a piecewise-linear 3-manifold, $g: K \rightarrow \operatorname{Int} M$ a topological embedding, and $A_{1}, A_{2}, \ldots, A_{k}$, a finite disjoint collection of tame arcs in $M-g(L)$ with each $\mathrm{Bd} A_{j} \subset M-g(K)$. Then, for given $\varepsilon>0$, there is a homeomorphism $h: M \rightarrow M$ such that each $h\left(A_{i}\right) \subset M-g(K)$ and $h$ is the identity on $g(L)$ and outside the e-neighborhood of a compact set in $g(K)-g(L)$.

Proof. We first take a barycentric subdivision of $K$ so fine that the $g$-image of each simplex of this subdivision lies in an open 3-cell in $M$ (this will not destroy the contraction hypothesis, by [15, Theorem 4]). We then use Theorem 1 and induction on the number of elementary expansions required (after the subdivision) to expand $L$ into $K$. The details are routine, and will be omitted.

COROLlaRY 1.2. Theorem 1 is valid when, in its statement, $E^{3}$ is everywhere replaced by an arbitrary piecewise-linear 3-manifold $M$ such that $B \subset$ Int $M$.

Proof. Let $C$ and $E$ have the same meaning as in the first paragraph of the proof of Theorem 1. Then there is a homeomorphism of $[0,1]^{a}$ onto $B$ under which $[0,1]^{q-1} \times[0,1 / 2]$ corresponds to the closure of $B-C$. The result then follows from Corollary 1.1 and the fact that some subdivision $K$ of $[0,1]^{q}$ contains some subdivision $L$ of $[0,1]^{q-1} \times[0,1 / 2]$ as a subcomplex, with $K$ contracting to $L$. 
COROLlaRY 1.3. Let $K$ be a finite complex, $L$ a subcomplex of $K$ such that $K$ contracts to $L$, and $g: K \rightarrow$ Int $M$ a homeomorphism, where $M$ is a piecewise-linear 3-manifold. Then, if $g(L)$ has the cube-with-handles property, so does $g(K)$.

Proof. We may assume that $M$ is a compact 3-manifold-with-boundary, and hence an absolute neighborhood retract. There exists $\eta>0$ such that any two mappings of $S^{1}$ into $M$ which differ by less than $\eta$ are homotopic in $M$. Let $U$ be a neighborhood of $g(K)$ in $M$ and $r$ a retraction of $U$ onto $g(K)$ such that $r$ differs from the inclusion: $U \rightarrow M$ by less than $\eta$.

We claim that $U$ is an orientable neighborhood of $g(K)$. For, any loop $f: S^{1} \rightarrow U$ is homotopic in $M$ to the loop $r f: S^{1} \rightarrow g(K)$, hence to a loop in $g(L)$, and hence is orientation-preserving.

We may now apply the criterion of Lemma 2 to $g(K)$. Let $\varepsilon>0$ and a polygonal arc $A$ be given such that $g(K) \cap \mathrm{Bd} A=\varnothing$. Since $g(L)$ has the cube-with-handles property, there is a homeomorphism $h_{1}: M \rightarrow M$ which is the identity outside the $\varepsilon$-neighborhood of $g(L)$ and such that $g(L) \cap h_{1}(A)=\varnothing$. By Corollary 1.1, there is a homeomorphism $h_{2}: M \rightarrow M$ which is the identity outside the $\varepsilon$-neighborhood of $g(K)$ and such that $g(K) \cap h_{2} h_{1}(A)=\varnothing$. Then $h=h_{2} h_{1}$ is the required homeomorphism.

REMARK. It follows from Corollary 1.3 and the corollary to Lemma 3, that collapsible complexes and cubes-with-handles have the cube-with-handles property under every embedding in the interior of a 3-manifold $M$. If $M$ is orientable, then every homeomorphic image in Int $M$ of a compact 2-manifold with nonempty boundary has the cube-with-handles property.

COROllary 1.4. Let $M$ be a piecewise-linear 3-manifold, and $X$ a collapsible complex topologically embedded in Int $M$. Then $X$ is cellular with respect to piecewiselinear cells in $M$ if and only if: for each open set $U$ of $M$ containing $X$, there is an open set $V$ of $M$ such that $X \subset V \subset U$ and each loop in $V-X$ is contractible in $U-X$.

Proof. This is immediate from the previous remark and [12, Theorem 1'].

\section{REFERENCES}

1. R. H. Bing, Locally tame sets are tame, Ann. of Math. 59 (1954), 145-158.

2. - A surface is tame if its complement is 1-ULC, Trans. Amer. Math. Soc. 101 (1961), 294-305.

3. - Each disk in $E^{3}$ contains a tame arc, Amer. J. Math. 84 (1962), 583-590.

4. - - Each disk in $E^{3}$ is pierced by a tame arc, Amer. J. Math. 84 (1962), 591-599.

5. - Approximating surfaces from the side, Ann. of Math. 77 (1963), 145-192.

6. _- Pushing a 2-sphere into its complement, Michigan Math. J. 11 (1964), 33-45.

7. - Improving the side approximation theorem, Trans. Amer. Math. Soc. 116 (1965), 511-525.

8. - Improving the intersections of lines and surfaces, (to appear)

9. P. H. Doyle, Unions of cell pairs in $E^{3}$, Pacific J. Math. 10 (1960), 521-524.

10. P. H. Doyle and J. G. Hocking, Some results on tame disks and spheres in $E^{3}$, Proc. Amer. Math. Soc. 11 (1960), 832-836. 
11. Joseph Martin, Tame arcs on disks, Proc. Amer. Math. Soc. 16 (1965), 131-133.

12. D. R. McMillan, Jr., A criterion for cellularity in a manifold, Ann. of Math. 79 (1964), 327-337.

13. E. E. Moise, Affine structures in 3-manifolds. V. The triangulation theorem and Hauptvermutung, Ann. of Math. 56 (1952), 96-114.

14. - Affine structures in 3-manifolds. VII. Disks which are pierced by intervals, Ann. of Math. 58 (1953), 403-408.

15. J. H. C. Whitehead, Simplicial spaces, nuclei, and m-groups, Proc. London Math. Soc. 45 (1939), 243-327.

The UNIVERSITY OF VIRGINIA,

Charlottesville, Virginia 